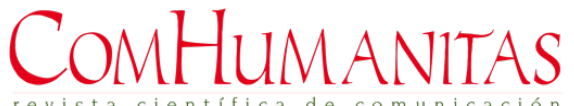

Revista ComHumanitas, ISSN: 1390-776X

\title{
Recordación de identidad visual de empresas ecuatorianas de alimentos y bebidas en Pichincha a través de línea gráfica establecida en Facebook: perspectiva del consumidor
}

\section{Visual identity remembrance of Ecuadorian food and beverages companies in Pichincha that use an established graphic line in Facebook: consumer perspective}

\section{Lembrança de identidade visual das empresas de alimentos e bebidas equatorianas em Pichincha que usam uma linha gráfica estabelecida no Facebook: perspectiva do consumidor}

\author{
Alejandro Javier Castillo Díaz ${ }^{1}$ \\ Instituto Tecnológico Superior Cordillera (Ecuador) \\ alejandro.castillo@cordillera.edu.ec \\ Jonathan Marcelo Vinueza Suárez ${ }^{2}$ \\ Instituto Tecnológico Superior Cordillera (Ecuador) \\ jonathan.vinueza@cordillera.edu.ec
}

Fecha de recepción: 29 de noviembre de 2018 Fecha de recepción evaluador: 25 de marzo de 2019

Fecha de recepción corrección: 26 de marzo de 2019

\footnotetext{
${ }^{1}$ Alejandro Javier Castillo Díaz es publicista y Magister en Dirección de Comunicación, con experiencia en agencias de publicidad y de marketing de empresas privadas. https://orcid.org/0000-0002-7132-6265

2 Jonathan Marcelo Vinueza Suárez es licenciado en Comunicación Social, con experiencia en Docencia, en Diseño Gráfico en el Instituto Tecnológico Superior Cordillera. https://orcid.org/0000-0001-6471-1344
} 


\title{
Resumen
}

El presente estudio demuestra los efectos de la utilización de línea gráfica en Facebook en los niveles de recordación de identidad visual de 111 bares y restaurantes ecuatorianos afiliados a la Cámara de Turismo de Pichincha, provincia de la región Andina de Ecuador. La finalidad, fue comprobar la relevancia de la tarea de diseñadores gráficos profesionales dentro de espacios digitales interactivos. Se clasificó a estos establecimientos en dos grupos, 65 que utilizan línea gráfica en esta red social y 46 que no lo hacen. Se realizaron 385 encuestas a clientes en cada grupo de establecimientos, 770 en total, lo que permitió establecer cuadros comparativos y plantear conclusiones. Se demostró que la utilización de línea gráfica en los 65 establecimientos del primer grupo, influye en los niveles de recordación de su identidad visual con respecto a los 46 establecimientos del segundo grupo. Por lo tanto, se visualiza la necesidad de crear canales que involucren a diseñadores gráficos expertos en construcción y administración de marcas en procesos de marketing digital por parte de empresas del sector turístico de la provincia de Pichincha.

Palabras clave: Identidad visual corporativa, marketing digital, redes sociales, diseño de marca, diseño gráfico, turismo Pichincha

\begin{abstract}
The present study demonstrates the effects of the use of graphic line on Facebook in the levels of visual identity recall of 111 Ecuadorian restaurants and bars affiliated to Pichincha Chamber of Tourism, province of the Andean region of Ecuaodor. The purpose was to verify the relevance of the task of professional graphic designers within interactive digital spaces. These establishments were classified into two groups, 65 that use graphic lines in this social network and 46 that do not. 385 surveys were done to customers in each group of establishments, 770 in total, that allowed to make comparative tables and conclusions. It was demonstrated that the use of graphic line in the 65 establishments of the first group influences in the levels of remembrance of their visual identity with respect to the 46 establishments of the second group. Therefore, it is concluded that it is necessary to create channels to involve expert graphic designers in the construction of the brand management of the digital marketing processes by tourism companies in the province of Pichincha.
\end{abstract}

Keywords: Corporate visual identity, digital marketing, social media, brand design, graphic design, Pichincha tourism

\section{Resumo}

O presente estudo demonstra os efeitos do uso da linha gráfica no Facebook nos níveis de recordação visual de identidade de 111 restaurantes e bares equatorianos afiliados à Câmara de Turismo de Pichincha, província da região andina de Ecuaodor. O objetivo foi verificar a relevância da tarefa dos designers gráficos profissionais dentro dos espaços digitais interativos. Esses estabelecimentos foram classificados em dois grupos, 65 que utilizam linhas gráficas nessa rede social e 46 que não. 385 inquéritos foram feitos aos 
clientes em cada grupo de estabelecimentos, 770 no total, que permitiram fazer tabelas comparativas e conclusões. Ficou demonstrado que o uso da linha gráfica nos 65 estabelecimentos do primeiro grupo influencia nos níveis de recordação de sua identidade visual em relação aos 46 estabelecimentos do segundo grupo. Portanto, conclui-se que é necessário criar canais para envolver designers gráficos especializados na construção da gestão da marca dos processos de marketing digital por empresas de turismo na província de Pichincha.

Palavras-chave: identidade visual corporativa, marketing digital, mídias sociais, design de marca, design gráfico, turismo em Pichincha

\section{Introducción}

Facebook es una plataforma que mantiene un crecimiento continuo en Ecuador. En enero de 2016, existían un total de 8.9 millones de usuarios ecuatorianos que tenían un perfil en esta red social (Del Alcazar, 2016). Apenas dos años después, el número de inscritos en Ecuador creció a un total de 12 millones, de los cuales 11 millones presentaban actividad frecuente mensual (Del Alcazar, 2018).

Estas cifras, han sido de conocimiento e interés para establecimientos que ofertan servicios turísticos en la provincia de Pichincha y que han visto a Facebook como el canal ideal para generar interacción con el público. En 2017, la Cámara de Turismo de Pichincha (CAPTUR) contaba con un total de 900 establecimientos afiliados a esta organización, de los cuales, 394 se encontraban en la categoría Alimentos y Bebidas (Cámara de Turismo de Pichincha, 2017). A través de la observación de cada uno de estos negocios se pudo determinar que 129 de ellos (32,74\%), son ecuatorianos y poseen una página en Facebook.

Salvador (Comunicación Personal, 25 de enero de 2018), Director Ejecutivo de CAPTUR, indica que los perfiles de los clientes de estos establecimientos pueden variar de acuerdo a su ubicación geográfica, categoría u horario, pero, en términos generales, la mayoría de ellos se encuentran en el rango de edad de 25 a 45 años debido a diversos factores como su actividad laboral o capacidad económica.

\section{Marco referencial}

Para Currás (2010), la identidad corporativa es una proyección o representación de una organización, a través de su simbología, comunicación o comportamiento, para dar importancia a ciertos elementos centrales de su identidad como son su cultura, sus valores o su alma. Además, complementa, que la identidad visual es la representación gráfica de la identidad corporativa de una organización y está conformada una serie de signos que pueden llegar en forma de logo, slogan o cualquier otra forma que puede ser captada e interpretada (Currás, 2010).

Hidalgo, Chiriboga y Mora (2015), plantearon que la identidad visual refleja la filosofía y los valores de una organización. Argumentaron, además, que dos elementos 
claves de la identidad visual son su logotipo, es decir la representación gráfica de su nombre y su isotipo, el elemento gráfico que representa a una institución sin hacer uso de las palabras.

Varias marcas han utilizado de manera exitosa a Facebook para la promoción y difusión de su identidad visual. Ruiz (2015), resaltó la importancia de Facebook para el éxito de Hawkers, empresa online dedicada a la fabricación de gafas de sol. En dicho proyecto, se destaca que gran parte del éxito de la compañía en esta red social se debió a la naturaleza de sus publicaciones y a las distintas formas de interacción que se generaron con el público.

En Ecuador, existe una creciente masificación de las redes sociales utilizadas por marcas con fines publicitarios, lo que hace que las empresas tengan que gestionar sus esfuerzos de posicionamiento en estos nuevos medios, manteniendo la base de sus signos de identidad, pero adaptándose a un lenguaje interactivo (Paladines, Granda, \& Velásquez, 2014). Jaramillo (2012), determinó que entre las empresas turísticas de la parroquia Mindo (atractivo turístico del noroccidente de la provincia de Pichincha) que habían utilizado el internet como medio fundamental de promoción de sus establecimientos, el 26,42\% prefería las redes sociales sobre otros medios digitales y, de ellas, el 66,67\% utilizaban a Facebook como la red social fundamental para difusión de eventos, paquetes y diversas actividades turísticas.

En abril de 2017, ejecutivos de la agencia de publicidad Castelvania S.A., bajo la aprobación de CAPTUR Pichincha, realizaron una serie visitas a establecimientos afiliados a la Cámara para elaborar un diagnóstico de cómo se estaban administrando sus páginas en Facebook. El objetivo de estas visitas, fue ofrecer asesoría para el manejo de su identidad visual y la elaboración de sus líneas de contenidos en esta red social (Erazo, 2018). En diciembre de 2017, Castelvania S.A. trabajaba con un total de 7 empresas afiliadas a la Cámara, de las cuales 4 se encontraban dentro de la categoría Alimentos y Bebidas. En esta gestión, se pudo inferir un aumento en los niveles de recordación de la identidad visual de estos establecimientos, pero sin el registro formal de datos que lo corrobore.

En este contexto, se planteó resaltar la labor de diseñadores gráficos expertos en diseño de identidad visual y la labor realizada por establecimientos turísticos de la provincia de Pichincha para generar contacto e interacción con sus públicos preferenciales. El objetivo fue el de comprobar la aplicación de una línea gráfica definida en Facebook y su repercusión en el recuerdo de la identidad visual por parte de los clientes de empresas ecuatorianas afiliadas a la Cámara de Turismo de Pichincha (categoría Alimentos y Bebidas).

Se buscó, por lo tanto, establecer y demostrar la necesidad que tienen las empresas del sector turístico ecuatoriano de manejar línea gráfica en sus publicaciones en Facebook para que no se encuentren en desventaja competitiva. Por otro lado, se pretende dotar al sector turístico de la provincia de Pichincha de herramientas de análisis que permitan tomar decisiones estratégicas en materia de Identidad Visual. Finalmente, se ha querido 
demostrar la importancia del diseño gráfico dentro de la gestión de espacios digitales interactivos para establecimientos afiliados a la Cámara.

\section{Metodología}

El objetivo de la identidad visual, es el de diferenciar a una organización de otras a través de un sistema conformado por signos y símbolos que la representen (Santamaría \& Osorio, 2014). Se establecieron tres parámetros básicos de la línea gráfica basados en los planteamientos de Bustamante (2012) que fueron: el logotipo, la consistencia en el manejo cromático y la utilización de una misma tipografía.

A continuación, se realizó una observación de los fanpage de Facebook de los 129 establecimientos ecuatorianos pertenecientes a la categoría Alimentos y Bebidas registrados en la página web de CAPTUR Pichincha (2017). A través de esta observación, se pudo determinar que 77 empresas cumplen con los tres parámetros básicos que implican la utilización de línea gráfica, mientras que 52 no lo hacen. Estos establecimientos fueron categorizados en 2 grupos A y B (Tabla 1):

Tabla 1: Categorización de grupos de establecimientos y muestra aleatoria

\begin{tabular}{llll}
\hline Código & $\begin{array}{l}\text { No. } \\
\text { empresas }\end{array}$ & $\begin{array}{l}\text { No. de establecimientos } \\
\text { de la muestra }\end{array}$ & Descripción \\
\hline Grupo A & 77 & 65 & $\begin{array}{l}\text { Establecimientos que sí utilizan línea gráfica en } \\
\text { Facebook }\end{array}$ \\
\hline Grupo B & 52 & 46 & $\begin{array}{l}\text { Establecimientos que no utilizan línea gráfica en } \\
\text { Facebook }\end{array}$ \\
\hline
\end{tabular}

Con el fin de realizar un estudio comparativo que permita observar cambios en los establecimientos en ambos grupos de establecimientos, se aplicaron encuestas de recordación de marca a cada uno de ellos. Para ello, se utilizó un muestreo aleatorio simple con un margen de error del 5\%. Se realizaron 385 encuestas en cada categoría de establecimientos con un nivel de confianza del 95\% ( $\mathrm{N}=770)$.

Los criterios de exclusión de los cuestionarios realizados en los establecimientos de ambos grupos fueron los encuestados que no entraban en el rango 25 a 45 años de edad y que no poseían una cuenta en Facebook. A los participantes que sí cumplieron con los requisitos, se les preguntó cuánto recuerdan, en escala ordinal de Likert de cinco niveles, los tres aspectos fundamentales de la línea gráfica del negocio en el que se encontraban, logotipo, cromática y tipografía. Se consideraron las siguientes variables cualitativas de opinión: 1 como nada reconocido y 5 totalmente reconocido. Adicionalmente, se les preguntó si Facebook pudo haber incidido en esos niveles de recordación en una escala del 1 al 5 siendo 1 totalmente en desacuerdo y 5 totalmente de acuerdo.

Para el análisis y procesamiento de los datos, las respuestas obtenidas en los cuestionarios fueron ingresadas en un archivo del software SPSS (Statistical Package for 
Social Sciences) Versión 23 y segmentadas de acuerdo a los dos grupos de estudio. La Tabla 2 muestra la organización de las variables analizadas:

Tabla 2: Descripción de variables analizadas

\begin{tabular}{ll}
\hline Código & Descripción \\
\hline A1 & Nivel de recordación del logotipo del Grupo A \\
\hline A2 & Nivel de recordación de la cromática del Grupo A \\
\hline A3 & Nivel de recordación de la tipografía del Grupo A \\
\hline A4 & Percepción de Facebook como la red social que influye sobre los niveles de recordación de \\
& los establecimientos en el Grupo A \\
\hline B1 & Nivel de recordación del logotipo del Grupo B \\
\hline B2 & Nivel de recordación de la cromática del Grupo B \\
\hline B3 & Nivel de recordación de la tipografía del Grupo B \\
\hline B4 & Percepción de Facebook como la red social que influye sobre los niveles de recordación de \\
&
\end{tabular}

Para garantizar la fiabilidad del estudio, se calculó el Alfa de Cronbach de los datos obtenidos de las tres primeras variables de los Grupos A $(\alpha=, 804)$ y B $(\alpha=, 779)$. Los resultados obtenidos indican coeficientes cercanos a $\alpha=, 8$ por lo que se puede, por lo tanto, garantizar que los resultados son fiables.

\section{Análisis de datos}

Se generó una prueba de normalidad de las variables A1, A2, A3, A4, B1, B2, B3 y B4 en el programa SPSS. Para ello, se tomaron en cuenta los resultados arrojados por el test de Kolmogorov - Smirnov usado para muestras superiores a 50 encuestados.

A continuación, se realizaron tablas comparativas de las medias, medianas, modas, deviaciones típicas y varianzas entre las variables A1-B1, A2-B2, A3-B3 y A4B4. Adicionalmente, se generaron tablas de frecuencias de todas las variables para descubrir cuáles fueron las respuestas más escogidas en cada caso. Finalmente, se utilizó la prueba no paramétrica de $U$ de Mann-Whitney para definir si la hipótesis de diferenciación de grupos se cumplió.

\section{Hallazgos}

A través de la prueba de normalidad de Kolmogorv-Smirnov, se comprobó que los datos no fueron normales para las variables de ambos grupos $(p<.001)$ (Tabla 3 ). 
Tabla 3: Prueba de normalidad Kolmogorov-Smirnov de las variables de ambos grupos

\begin{tabular}{llll}
\hline Grupo & Estadístico & Gl & Sig. \\
\hline A1 &, 208 & 385 &, 000 \\
\hline A2 & 168 & 385 &, 000 \\
\hline A3 & 183 & 385 &, 000 \\
\hline A4 & 163 & 385 &, 000 \\
\hline B2 & 199 & 385 &, 000 \\
\hline B3 & 189 & 385 &, 000 \\
\hline B4 &, 193 & 385 &, 000 \\
\hline
\end{tabular}

A continuación, se establecen datos comparativos de media, mediana, moda, desviación típica y varianza las variables Logotipo, Cromática y Tipografía de los grupos A y B (Tabla 4)

Tabla 4: Estadísticos descriptivos entre pares de grupos analizados

\begin{tabular}{lllllll}
\hline & $\begin{array}{c}\text { Grupos } \\
\text { A1 vs. B1 }\end{array}$ & & $\begin{array}{c}\text { Grupos } \\
\text { A2 vs. B2 }\end{array}$ & & A3 vs. B3 & Arupos \\
& A1 & B1 & A2 & B2 & A3 & B3 \\
\hline Media & 3,61 & 3,19 & 3,04 & 2,49 & 3,21 & 2,56 \\
\hline Mediana & 4,00 & 3,00 & 3,00 & 2,00 & 3,00 & 3,00 \\
\hline Moda & 4 & 4 & 3 & 2 & 4 & 3 \\
\hline Desv. típ. & 1,047 & 1,242 & 1,115 & 1,159 & 1,176 & 1,158 \\
\hline Varianza & 1,097 & 1,543 & 1,243 & 1,344 & 1,384 & 1,341 \\
\hline
\end{tabular}

En todos los casos, la media de las variables del grupo A son superiores a las medias de las variables del grupo B en rangos que van de 0,42/5 (Logotipo) a 0,65/5 (Tipografía).

Por otro lado, la mediana y la moda no siguen una misma línea, aunque en ninguno de los casos los valores de estas medidas son superiores en las variables del grupo B con respecto a las variables del grupo A. Esto confirma que no existe una distribución normal.

Se aprecia, también, que los datos de desviación típica y varianza no siguen una misma tendencia por lo que no se podría determinar cambios en la medida de dispersión con respecto a la media entre las variables del grupo A con respecto a las variables del grupo B.

A continuación, se exponen tablas y diagramas de caja comparativos entre las variables A4 y B4 que tienen que ver con la percepción de que Facebook pudo haber influido en las respuestas a las tres primeras preguntas. 
Tabla 5: Estadísticos descriptivos entre variables A4 y B4

\begin{tabular}{lll}
\hline & A4 & B4 \\
\hline Media & 3,14 & 3,15 \\
\hline Mediana & 3,00 & 3,00 \\
\hline Moda & 3 & 4 \\
\hline Desv. típ. & 1,288 & 1,392 \\
\hline Varianza & 1,659 & 1,939 \\
\hline
\end{tabular}

Las variables A4 y B4 presentan resultados casi idénticos de media y mediana y resultados bastante similares de moda, desviación típica y varianza. Para poder apreciar dónde se registraron la mayor parte de las respuestas de las variables entre las cuales se realiza la comparación, se presentan a continuación tablas de frecuencias de las tres primeras variables (Tablas $6,7,8$ y 9):

Tabla 6: Escala cualitativa de reconocimiento, frecuencia y porcentaje de las variables A1 y B1

\begin{tabular}{lllll}
\hline & Grupo A1 & Grupo B1 & \\
\hline $\begin{array}{l}\text { Escala } \\
\text { cualitativa }\end{array}$ & Frecuencia & Porcentaje (\%) & Frecuencia & Porcentaje (\%) \\
\hline $\begin{array}{llll}\text { Nada reconocido } \\
\text { Poco reconocido }\end{array}$ & 14 & 3,6 & 53 & 13,8 \\
$\begin{array}{l}\text { Aceptablemente } \\
\text { reconocido }\end{array}$ & 116 & 30,1 & 51 & 13,2 \\
$\begin{array}{l}\text { Bastante } \\
\text { reconocido }\end{array}$ & 132 & 34,3 & 105 & 27,3 \\
$\begin{array}{l}\text { Totalmente } \\
\text { reconocido }\end{array}$ & 85 & 22,1 & 120 & 31,2 \\
Total & 385 & 100 & 56 & 14,5 \\
\hline
\end{tabular}

La comparación de frecuencias de las variables A1 y B1, que tienen que ver con la recordación del logotipo en los establecimientos, prueba que existen resultados bastante favorables para los establecimientos del grupo A, ya que el 56,4\% de ellos son bastante o totalmente reconocidos en comparación con un 45,7\% del grupo B. Por su parte, existe un $13,8 \%$ de encuestados que desconocen por completo al logotipo del establecimiento que se encontraban en el grupo B, mientras que apenas el 3,6\% de encuestados del grupo A consideran al logotipo nada reconocido. 
Tabla 7: Escala cualitativa de reconocimiento, frecuencia y porcentaje de las variables A2 y B2

\begin{tabular}{lllll}
\hline \multicolumn{3}{l}{ Grupo A2 } & Grupo B2 \\
\hline $\begin{array}{l}\text { Escala } \\
\text { cualitativa }\end{array}$ & Frecuencia & Porcentaje (\%) & Frecuencia & Porcentaje (\%) \\
\hline Nada reconocido & 36 & 9,4 & 89 & 23,1 \\
$\begin{array}{l}\text { Poco reconocido } \\
86\end{array}$ & 22,3 & 113 & 29,4 \\
$\begin{array}{l}\text { Aceptablemente } \\
\text { reconocido }\end{array}$ & 126 & 32,7 & 112 & 29,1 \\
$\begin{array}{l}\text { Bastante } \\
\text { reconocido }\end{array}$ & 100 & 26,0 & & \\
$\begin{array}{l}\text { Totalmente } \\
\text { reconocido }\end{array}$ & 37 & 9,6 & 46 & 11,9 \\
Total & 385 & 100,0 & 25 & 6,5 \\
\hline
\end{tabular}

Con respecto a la variable cromática, se puede apreciar que existen menores niveles de recordación con respecto a los que existían en la primera variable para ambos casos, pero también se pueden extraer datos que favorecen los niveles de recordación de los colores corporativos de los establecimientos del grupo A con respecto a los establecimientos del grupo B. Más de la mitad de los encuestados (el 52,5\%), indicó que los colores de los establecimientos del grupo B eran poco o nada reconocidos, una cifra que difiere de la obtenida entre los encuestados de los establecimientos del grupo A $(31,7 \%)$. Por otro lado, el $35,6 \%$ de los encuestados del grupo A opinaron que la cromática de sus establecimientos era bastante o totalmente reconocida, con respecto a un $18,4 \%$ de los encuestados del grupo B.

Tabla 8: Escala cualitativa de reconocimiento, frecuencia y porcentaje de las variables A3 y B3

\begin{tabular}{lllll}
\hline \multicolumn{3}{l}{ Grupo A3 } & Grupo B3 & \\
\hline $\begin{array}{l}\text { Escala } \\
\text { cualitativa }\end{array}$ & Frecuencia & Porcentaje (\%) & Frecuencia & Porcentaje (\%) \\
\hline Nada reconocido & 35 & 9,1 & 92 & 23,9 \\
$\begin{array}{l}\text { Poco reconocido } \\
73\end{array}$ & 19,0 & 83 & 21,6 \\
$\begin{array}{l}\text { Aceptablemente } \\
\text { reconocido }\end{array}$ & 110 & 28,6 & 132 & 34,3 \\
$\begin{array}{l}\text { Bastante } \\
\text { reconocido }\end{array}$ & 111 & 28,8 & & \\
$\begin{array}{l}\text { Totalmente } \\
\text { reconocido }\end{array}$ & 56 & 14,5 & 58 & 15,1 \\
Total & 385 & 100,0 & 20 & 5,2 \\
\hline
\end{tabular}


La variable tipografía sigue la misma tendencia que las variables logotipo y cromática y se puede apreciar una mayor inclinación hacia las respuestas 1 y 5 que en las otras variables. Un $23,9 \%$ de los encuestados del grupo B, afirma desconocer por completo la tipografía que se usa en el establecimiento que se encontraban, mientras que apenas un 5,2\% afirmaba que era totalmente reconocida por ellos. Esto contrasta con los porcentajes obtenidos entre los encuestados del grupo A, que propiciaron valores de 9,1 y $14,5 \%$ respectivamente.

Tabla 9 Escala cualitativa de reconocimiento, frecuencia y porcentaje de las variables A4 y B4

\begin{tabular}{lllll}
\hline & Grupo A4 & Grupo B4 & \\
\hline $\begin{array}{l}\text { Escala } \\
\text { cualitativa }\end{array}$ & Frecuencia & Porcentaje (\%) & Frecuencia & Porcentaje (\%) \\
\hline Nada reconocido & 50 & 13,0 & 69 & 17,9 \\
Poco reconocido & 75 & 19,5 & 62 & 16,1 \\
$\begin{array}{l}\text { Aceptablemente } \\
\text { reconocido }\end{array}$ & 100 & 26,0 & 76 & 19,7 \\
$\begin{array}{l}\text { Bastante } \\
\text { reconocido }\end{array}$ & 90 & 23,4 & 99 & 25,7 \\
$\begin{array}{l}\text { Totalmente } \\
\text { reconocido }\end{array}$ & 70 & 18,2 & 79 & 20,5 \\
Total & 385 & 100,0 & 385 & 100,0 \\
\hline
\end{tabular}

La percepción de que Facebook pudo haber incidido en las respuestas de las 3 primeras variables, sigue una tendencia distinta en este caso. Se pueden observar ciertas diferencias. En el grupo A, por ejemplo, el mayor porcentaje de las respuestas $(49,4 \%)$ se ubica en las respuestas 3 y 4, ni lo uno ni lo otro y bastante de acuerdo. Por su parte, los encuestados del grupo B, favorecieron sobre todo a las preguntas 4 y 5 , bastante de acuerdo y totalmente de acuerdo (46,2\%). Adicionalmente, un 17,9\% de los encuestados del grupo B, expresó que estaba totalmente en desacuerdo con la premisa, con respecto a un $13 \%$ del grupo A.

Se realizó una prueba no paramétrica para visualizar si existen diferencias entre los dos grupos. Por tanto, se presenta a continuación la prueba de U de Mann-Whitney para variables independientes. Para ello, se plantearon dos posibles hipótesis:

- $\mathrm{H} 0=$ Los niveles de recordación de identidad visual de las empresas de la categoría Alimentos y Bebidas afiliadas a CAPTUR son los mismos entre todos los tipos de establecimiento

- $\quad \mathrm{H} 1=$ Los niveles de recordación de identidad visual de las empresas de la categoría Alimentos y Bebida, afiliadas a CAPTUR son distintos entre los dos grupos de establecimiento afiliados a la Cámara. 
Tabla 10: Prueba U de Mann-Whitney

\begin{tabular}{lllll}
\hline & Logotipo & Cromática & Tipografía & Facebook \\
\hline U de Mann-Whitney & 60864,000 & 54119,500 & 52063,500 & 73395,000 \\
\hline Sig. asintót. (bilateral) &, 000 &, 000 &, 000 &, 812 \\
\hline
\end{tabular}

La significación asintótica bilateral es menor a $.005(p=.000)$ para las tres primeras variables, con lo que queda rechazada la hipótesis nula. Se acepta, entonces, que los niveles de recordación de identidad visual son distintos en los dos grupos de establecimientos de Alimentos y Bebidas afiliados a la Cámara de Turismo de Pichincha.

\section{Discusión}

El término recordación sintetiza todo lo que asocia o recuerda la mente del consumidor a la hora de identificarse con una empresa (Rodríguez, 2016). Lograr que una marca sea recordada, es uno de los objetivos primarios de la publicidad, puesto que facilita la fidelidad por parte de los públicos de interés hacia un producto y propicia su compra repetitiva (López, Sandoval, \& Cortés, 2010). La recordación de marca en anuncios publicitarios, es determinada por un conjunto de factores que son propios de su estructura, como su diseño, color o posición (Torres \& Muñoz, 2006).

El presente estudio, partió de la hipótesis de que la utilización de una línea gráfica definida en Facebook ayuda a mejorar los niveles de recordación de identidad visual de establecimientos afiliados a la Cámara de Turismo de Pichincha (Categoría Alimentos y Bebidas). La observación realizada a estos establecimientos arrojó que, de las 129 empresas ecuatorianas que pertenecen a esta categoría y que poseen una página en Facebook, 52 de ellas no manejan línea gráfica en esta red social. Al conocer ya, que la hipótesis nula fue descartada, se acepta que esos 52 establecimientos se encuentran en desventaja competitiva y requieren soporte de profesionales del diseño gráfico para estructurar su identidad visual en Facebook y así no perder espacio en la mente y el corazón de sus consumidores (Correa \& Duque, 2012).

Además, fueron analizados 129 de los 394 establecimientos afiliados a CAPTUR (Cámara de Turismo de Pichincha, 2017) por ser los que cumplían con los requerimientos de ser ecuatorianos y poseer una página en Facebook. Esto representa una minoría y, por tanto, se debe potenciar mejoras de visibilidad de marca entre los establecimientos que no manejan esta herramienta digital.

Las variables logotipo, cromática y tipografía reflejan niveles de recordación más altos entre los grupos de establecimientos que manejan línea gráfica en Facebook en comparación con los que no lo hacen (Tablas 4), pero la percepción general es que Facebook influyó de la misma manera en ambos grupos (Tabla 5). Por consecuencia, lo que genera estas diferencias, es la utilización de línea gráfica. Al ser la recordación un factor de vital importancia para la sostenibilidad a largo plazo de una empresa por su estrecha relación con las emociones de las personas (Rodríguez, 2016), los bares y 
restaurantes de la provincia de Pichincha, deben manejar una línea gráfica definida en esta red social para verse favorecidos por los efectos de la recordación de su marca.

De los tres elementos fundamentales que conforman la línea gráfica de una marca, logotipo, cromática y tipografía (Bustamante, 2012), fue el logotipo el que obtuvo una media de valoración más alta en los niveles de recordación de los establecimientos que manejan línea gráfica en Facebook (Tabla 4) y el obtuvo el porcentaje más alto como elemento totalmente reconocido con respecto a las otras variables (Tabla 6). Esto denota que los futuros diseñadores ecuatorianos que quieran ejercer exitosamente como profesionales de la identidad visual en Facebook deberán, prioritariamente, estar capacitados para desarrollar logos sean fáciles de reconocer y recordar por parte de los públicos que los observen en esta red social. Además, se conceptualiza la importancia de la línea gráfica como la base de la identidad visual de una empresa.

Mediante la prueba U de Mann-Whitney (Tabla 10), se comprobó exitosamente la aplicación de una línea gráfica definida en Facebook y su repercusión en el recuerdo de la identidad visual por parte de los clientes de empresas ecuatorianas afiliadas a la Cámara de Turismo de Pichincha (categoría Alimentos y Bebidas). La aceptación de la hipótesis, complementa de manera exitosa los estudios de Paladines, Granda y Velásquez (2014) y de Jaramillo (2012) que ya habían considerado a las redes sociales como canales poderosos para la difusión y posicionamiento de marcas a través de medios interactivos, pero no contemplaban, todavía, una relación con la práctica del diseño gráfico. Ante estos resultados, se pretende una evidencia más de la existencia de una interacción entre el estudio del marketing digital y el estudio del diseño gráfico, que deberán estar vinculados para lograr resultados de recordación óptimos por parte de empresas que apliquen criterios de línea gráfica en esta red social. Resulta necesario incorporar la labor y el conocimiento de un diseñador gráfico capacitado en la generación de procesos de marketing digital para garantizar su inserción en las compañías de diferentes sectores.

Al estar aprobada la hipótesis, se generan, también, nuevas necesidades para el desarrollo práctico del diseño gráfico, especialmente dentro de lo que tiene que ver con el desarrollo de identidad visual. Para la presente investigación, se han analizado estudios de caso como el expuesto por Bustamante (2012), o el expuesto por Hidalgo, Chiriboga y Mora (2015). En ninguno de estos casos se planteó la utilización de las marcas gráficas estudiadas en Facebook. Se sugiere la creación de futuros manuales que deberán dar pautas de la correcta utilización de logotipo, cromática y tipografía de una marca dentro de esta red social. Incluso, se plantea la necesidad de generar manuales específicos que definan los lineamientos de estos tres elementos de línea gráfica de una marca en Facebook, lo que incentivará al crecimiento de la industria del diseño en este nuevo campo.

Los diseñadores gráficos independientes pueden verse favorecidos por los resultados presentados, al encontrar en el desarrollo de marcas en Facebook una nueva alternativa laboral. Además, empresas que actualmente estén prestando servicios dentro de este campo, requerirán de la participación de expertos en área. Resulta, por tanto, 
importante que existan planes de capacitación y preparación de futuros profesionales del diseño que posean un mayor entendimiento de las redes sociales y de su alcance para la recordación de identidad visual de establecimientos turísticos.

\section{Conclusiones}

- Con la presentación de resultados del presente estudio, se concluye que:

- Los elementos que forman parte de la línea gráfica de una compañía son su logotipo, cromática y tipografía deben presentarse de manera consistente en publicaciones en Facebook para que ayuden a mejorar los niveles de recordación de la identidad visual de sus organizaciones.

- La recordación de identidad visual es un elemento de vital importancia que afecta, sobre todo, a la fidelidad de clientes hacia una organización.

- Existen 52 establecimientos pertenecientes a la categoría Alimentos y Bebidas afiliados a CAPTUR Pichincha que no manejan línea gráfica en Facebook y, por lo tanto, sus distintivos visuales tienen un menor nivel de recordación con respecto a los 77 establecimientos que sí lo hacen.

- Se requiere mayor vinculación de diseñadores gráficos capacitados en el diseño y desarrollo de identidad visual como parte de los procesos de creación de publicaciones en Facebook por parte de empresas del sector turístico ecuatoriano.

○ Los diseñadores gráficos ecuatorianos deben capacitarse en manejo de identidad visual de marcas en Facebook y contemplar un adecuado manejo de línea gráfica dentro de esta red social al momento del desarrollo de Manuales de Identidad Visual Corporativa para las empresas para las que estén prestando servicios.

\section{Notas}

\footnotetext{
${ }^{1}$ Alejandro Javier Castillo Díaz, Publicista y Magister en Dirección de Comunicación, con 13 años de experiencia en agencias de publicidad y departamentos de marketing de empresas privadas. Propietario de Castelvania S.A., agencia especializada en manejo de marcas en espacios web 2.0. Cuenta con seis semestres de experiencia en la Docencia Superior de materias como Marketing, Publicidad y Comunicación, colaborando también como Tutor y Lector de proyectos de grado, como Vocal en tribunales de defensa y como Jurado de Producción Artística. https://orcid.org/0000-0002-7132-6265
}

2 Jonathan Marcelo Vinueza Suárez, Licenciado en Comunicación Social, con cinco semestres de experiencia en Docencia, en la carrera de Diseño Gráfico en el Instituto Tecnológico Superior Cordillera. Ha trabajado en Wepa Music Records como Diseñador Multimedia. Actualmente inscrito en la Maestría de Diseño Gráfico Digital en la Universidad de la Rioja (UNIR-España). https://orcid.org/0000-0001-6471$\underline{1344}$ 


\section{Bibliografía}

Bustamante, A. (21 de Noviembre de 2012). Diseño de marca gráfica y creación del manual de identidad visual corporativa del proyecto "Cali de noche". Proyecto de grado para optar al título de Diseñador de la Comunicación Gráfica. (B. Roa, Ed.) Santiago de Cail, Colombia: Universidad Autónoma de Occidente. Recuperado el 1 de Junio de 2018, de Repositorio Institucional UAO: http://red.uao.edu.co/handle/10614/7836

Cámara de Turismo de Pichincha. (2017). Socios Activos 2017. Recuperado el 15 de Marzo de 2018, de CAPTUR: http://captur.travel/

Correa, C., \& Duque, E. (2012). Evaluación del concepto de valor para el consumidor en el marketing y el diseño. Una revisión conceptual. Criterio Libre, 10(17), 193 216. doi:ISSN 1900-0642

Currás, R. (2010). Identidad e imagen corporativas: revisión conceptual e interrelación. Teoría y Praxis(7), 9-34. Recuperado el 1 de Abril de 2018, de http://www.redalyc.org/html/4561/456145285002/

Del Alcazar, J. P. (30 de Enero de 2016). Estadísticas Facebook Ecuador. Recuperado el 1 de Marzo de 2018, de Formación Gerencial: http://blog.formaciongerencial.com/estadisticasfacebookecuador/

Del Alcazar, J. P. (Abril de 2018). Ecuador: Cifras digitales, perfil de consumidor y ranking de Sitios Web. Recuperado el 30 de Abril de 2018, de Formación Gerencial: http://blog.formaciongerencial.com/ecuador-cifras-digitales-perfil-deconsumidor-y-ranking-sitios-web/

Erazo, D. (25 de Enero de 2018). Entrevista de A. Castillo. Los clientes de Castelvania en 2017

Facebook. (2018). Facebook para empresas. Recuperado el 1 de Abril de 2018, de Facebook: https://www.facebook.com/business/

Hidalgo, J., Chiriboga, E., \& Mora, F. (2015). Estudio de caso: el remozamiento de la identidad visual corporativa del Banco Guayaquil en Ecuador. Razón y Palabra, 19(89), 346-362. Recuperado el 1 de Marzo de 2018, de http://www.revistarazonypalabra.org/index.php/ryp/article/view/246/284

Jaramillo, M. (Abril de 2012). Análisis del social media marketing en las principales redes sociales como My Space, Facebook, Hi5 y su impacto en la promoción turística de la Parroquia Mindo durante el periodo 2008-2010. Recuperado el 15 de Junio de 2018, de Repositorio digital UPS: https://dspace.ups.edu.ec/handle/123456789/3304

López, D., Sandoval, M., \& Cortés, O. (2010). Relación entre los niveles de TRP's, las medidas de recordación, preferencia de marca y la conducta de compra en 
consumidores colombianos. Psicología desde el Caribe(25), 31-55. Recuperado el 28 de Septiembre de 2019, de http://www.redalyc.org/html/213/21315106003/

Paladines, F., Granda, C., \& Velásquez, A. (Junio de 2014). La marca ecuatoriana y su gestión en redes sociales. Razón y Palabra(86). Recuperado el 1 de Abril de 2018, de http://www.redalyc.org/html/1995/199531505033/

Rodríguez, F. (5 de Diciembre de 2016). Recordación de Marca. Recuperado el 15 de Febrero de 2018, de Grupo Franja: https:/grupofranja.com/index.php/negocios/item/1517-recordacion-de-marca

Ruiz, B. (2015). Fenómeno Hawkers: caso de éxito en Facebook. Trabajo de fin de grado. Titulación de Periodismo. (R. Cifuentes, Ed.) Elche, Alicante, España: Universidad Miguel Hernández. Facultad de Ciencias Sociales y Jurídicas. $\begin{array}{llllll}\text { Recuperado el } 1 \text { de } & \text { Marzo de }\end{array}$ http://dspace.umh.es/bitstream/11000/3665/1/RUIZ\%20MIRALLES\%20BEAT RIZ.pdf

Salvador, A. (25 de Enero de 2018). Entrevista de A. Castillo. Los clientes potenciales de los establecimientos de Alimentos y Bebidas afiliados a CAPTUR.

Santamaría, J., \& Osorio, K. (8 de Mayo de 2014). Estudio del impacto de una identidad gráfica de las empresas artesanales salvadoreñas: diseño aplicado a la identidad gráfica de vinos artesanales Carranza de San Pedro Nonualco, Departamento de La Paz, 2013. Recuperado el 1 de Julio de 2018, de Eprints, repository software: http://ri.ues.edu.sv/5662/

Torres, E., \& Muñoz, J. (2006). Publicidad exterior: Estudio exploratorio de recordación de marca y motivación de compra. Recuperado el 15 de Junio de 2018, de Revista Venezola de Gerencia: http:/www.scielo.org.ve/scielo.php?pid=S131599842006000400005\&script=sci_arttext 